\title{
Presentación y desenlace del manejo conservador en obstrucción intestinal por bridas en el Cauca
}

\section{Presentation and outcome of conservative management of intestinal obstruction caused by adhesions in Cauca}

Anuar Armando Idrobo, ${ }^{1}$ (D) Beatriz Eugenia Bastidas, ${ }^{2}$ (D) Lina Marcela Yasno, ${ }^{3}$ (D) Andrés Leonardo Vargas, ${ }^{4}$ (D) Ángela María Merchán-Galvis. ${ }^{{ }^{*}}$ (B)

\author{
Gacceso abierto \\ Citación: \\ Idrobo AA, Bastidas BE, Yasno LM, Vargas AL, \\ Merchán-Galvis AM. Presentación y desenlace \\ del manejo conservador en obstrucción \\ intestinal por bridas en el Cauca. Rev Colomb \\ Gastroenterol. 2020;35(4):447-454. https://doi. \\ org/10.22516/25007440.517
}

Cirujano General, Fellow de Cirugía Pediátrica, Universidad del Valle. Cali, Colombia.

2 Médica docente, Especialista en Salud Familiar, Universidad del Cauca. Popayán, Colombia.

Médica, Magíster en Salud Ocupacional, Clínica La Estancia. Popayán, Colombia.

4 Médico, Hospital Susana López de Valencia, Popayán, Colombia.

5 Médica docente, Magíster en Epidemiología Clínica, Universidad del Cauca. Popayán, Colombia.

*Correspondencia: Ángela María Merchán-Galvis angelamerchan@unicauca.edu.co

Fecha recibido: $\quad 29 / 02 / 20$

Fecha aceptado: 10/08/20

\begin{abstract}
Resumen
Introducción: la obstrucción intestinal por bridas es responsable del $25 \%$ de las consultas a urgencias por dolor abdominal agudo, generando un alto riesgo de lesión iatrogénica por reintervenciones de urgencia. Una alternativa para determinar la necesidad de manejo quirúrgico sería el manejo médico conservador inicial y evaluar su respuesta en un período de 12-72 horas. Objetivo: describir los desenlaces clínicos de los pacientes adultos con obstrucción intestinal por bridas en un hospital de tercer nivel y que recibieron manejo médico no quirúrgico al ingreso. Metodología: estudio observacional tipo cohorte, prospectivo, de los pacientes atendidos con tratamiento conservador durante el período 2012-2013 por obstrucción intestinal. Se recogió información sociodemográfica y clínica de los pacientes, así como seguimiento hasta el alta hospitalaria. Para el análisis se empleó estadística descriptiva y se analizó con SPSS V.15. Resultados: el 58,9\% de los casos eran masculinos con edad media de 55,2 años, el 57,1\% tenía comorbilidades en las que predominaron las enfermedades cardiovasculares y $91,1 \%$ tenía antecedentes quirúrgicos, la mitad de ellos por procesos inflamatorios. Los principales síntomas fueron emesis y distención abdominal. La respuesta al tratamiento conservador fue de $80,4 \%$; del 19,6\% que requirió manejo quirúrgico, en el $81,8 \%$ de los casos el hallazgo intraoperatorio fue de obstrucción intestinal por bridas. El tiempo medio de hospitalización fue de 5,5 días y durante el estudio solo un paciente falleció. Conclusión: el manejo médico conservador debe ser considerado como el tratamiento inicial de esta entidad por su alta tasa de respuesta, disminución de complicaciones posoperatorias y estancia hospitalaria.
\end{abstract}

\section{Palabras clave}

Obstrucción intestinal, peritoneo, motilidad gastrointestinal, signos y síntomas.

\begin{abstract}
Introduction: Intestinal obstruction by adhesions accounts for $25 \%$ of consultations to the emergency department associated with acute abdominal pain, generating a high risk of iatrogenic injury by emergency reinterventions. An alternative to determine the need for surgical treatment is providing conservative medical management first and then assessing patients' response within 12-72 hours. Objective: To describe the clinical outcomes of adult patients with bowel obstruction caused by adhesions treated at a tertiary care hospital who received non-surgical medical treatment on admission. Methodology: Observational, prospective cohort study on patients treated conservatively during the period 2012-2013 for intestinal obstruction. Sociodemographic and clinical information was collected from patients, and follow-up was done until discharge. The analysis was carried out using descriptive statistics in SPSS v15. Results: $58.9 \%$ of the cases occurred in men, and the average age was 55.2 years. $57.1 \%$ of the patients had comorbidities predominantly associated with cardiovascular diseases, and $91.1 \%$ had previously undergone a surgical procedure, half of them due to inflammatory processes. The most common symptoms were emesis and abdominal distention. The response to conservative treatment was $80.4 \%$ of the $19.6 \%$ that required surgical management. In $81.8 \%$ of the cases, intestinal obstruction caused by adhesions was the main intraoperative finding. The average time of hospitalization was 5.5 days, and during the study only one patient died. Conclusion: Conservative medical management should be considered as the initial treatment for this entity due to its high response rate, reduction of postoperative complications, and shorter hospital stay.
\end{abstract}

Keywords

Intestinal obstruction, Peritoneum, Gastrointestinal motility, Signs and symptoms. 


\section{INTRODUCCIÓN}

Las adherencias peritoneales o bridas son el tejido cicatricial entre órganos abdominales, mesenterio y la pared abdominal que aparece después del trauma (1), generalmente por procedimientos quirúrgicos (2-6), esta entidad es responsable del $60 \%-70 \%$ de los cuadros de obstrucción intestinal $(2,6,7)$, definida como la detención del tránsito o de la progresión del contenido intestinal debido a una causa mecánica (8-10).

Aunque se ha estudiado ampliamente en pacientes ginecológicas (11), en quienes se ha determinado como factor de riesgo para infertilidad (12-16), no se conocen los factores determinantes para la presentación de esta patología. Se han propuesto como factores relacionados la técnica quirúrgica, el área a incidir y la cantidad de procedimientos previos (5, 17-20); sin embargo, no se ha encontrado una relación directa con el origen de esta patología (6).

La obstrucción intestinal por bridas (OIB) es responsable del $25 \%$ de las consultas a urgencias por dolor abdominal agudo (21), generando un alto riesgo de lesión iatrogénica por reintervenciones de urgencia $(22,23)$, que no siempre solucionan el cuadro. Por tanto, debe tenerse en cuenta la alternativa de manejo médico conservador inicial y evaluar su respuesta en un período de 72 horas para determinar la necesidad de manejo quirúrgico (24).

En Colombia, específicamente en el Cauca, no se tienen estadísticas sobre la presentación de la OIB, por lo que se buscó describir la presentación y los desenlaces clínicos de los pacientes adultos con este diagnóstico en el Hospital Universitario San José (HUSJ) y que recibieron manejo médico no quirúrgico al ingreso durante el período 2012-2013.

\section{MATERIALES Y MÉTODOS}

Este fue un estudio descriptivo tipo cohorte prospectiva de los pacientes que ingresaron con diagnóstico de OIB al HUSJ de Popayán y que recibieron manejo médico al ingreso durante el período de octubre de 2012 a septiembre de 2013.

Fueron criterios de inclusión haber ingresado al servicio de urgencias con cuadro sugestivo de OIB, ser candidato a manejo médico de su patología obstructiva y que aceptara participar en el estudio mediante la firma del consentimiento informado. Los criterios de exclusión fueron ser remitido a otra institución o manifestar su deseo de retirarse del proyecto durante el seguimiento.

Para la realización de este estudio, se utilizó un muestreo no probabilístico por conveniencia de todos los pacientes que fueron atendidos durante el período de recolección de la información. Se recogió información sociodemográfica y clínica de los pacientes relacionada con la presentación de la obstrucción intestinal y su respuesta al tratamiento (antecedentes quirúrgicos, signos y síntomas, duración del cuadro, respuesta al manejo médico, necesidad de cirugía y complicaciones). Se realizó la revisión de las historias clínicas y seguimiento a la respuesta clínica del manejo médico a las 12,24 y 48 horas hasta el egreso.

Para el análisis de las variables se utilizó estadística descriptiva, calculando medidas de tendencia central y de dispersión para las cuantitativas, así como frecuencias y proporciones para las variables categóricas. Se decidió comparar los desenlaces entre los pacientes que respondieron al manejo conservador y los que finalmente fueron llevados a cirugía, para ello se empleó la prueba de chi cuadrado $\left(\chi^{2}\right)$. Los datos fueron registrados y procesados en el programa estadístico SPSS V.15. Para la realización de este trabajo se contó con el aval del comité de ética del HUSJ y se tuvieron en cuenta las normas de investigación en seres humanos establecidas en la Declaración de Helsinki y la Resolución n. ${ }^{\circ} 008430$ de 1993 del Ministerio de Salud.

\section{RESULTADOS}

En el período de estudio ingresaron al HUSJ 78 pacientes con diagnóstico de OIB, de los cuales 15 fueron remitidos a otras instituciones y 7 se negaron a participar en el estudio, por lo que se contó con una muestra de 56 pacientes. De la población incluida $(\mathrm{n}=56), 58,9 \%(\mathrm{n}=33)$ era de sexo masculino, con una edad media de 55,2 años (rango: 19-85; desviación estándar [DE]: 20,2). Respecto a su procedencia, un 60,7 \% $(\mathrm{n}=34)$ pertenecía al área urbana; el 46,4\% tenía un nivel académico de bachiller $(\mathrm{n}=26)$, un $57,1 \%$ $(\mathrm{n}=32)$ pertenecía al estrato socioeconómico 1 y $55,4 \%$ estaba vinculado al régimen subsidiado $(\mathrm{n}=31)$. En la Tabla 1 se describen las características sociodemográficas de los pacientes del estudio.

Entre los antecedentes quirúrgicos se encontró que el $91,1 \%(\mathrm{n}=51)$ de los pacientes tuvo cirugía abdominal, en el $48 \%(n=24)$ de los casos por procesos inflamatorios. El $19,6 \%(n=11)$ de los pacientes tenía antecedentes de cirugía pélvica, entre ellas cesáreas, prostatectomía y anexo/ histerectomía. La media de los procedimientos quirúrgicos fue de una cirugía (rango de 0 a 8 ; DE: 1,2 ) y el tiempo de estos antecedentes quirúrgicos fue, en el 30,4\%, en un período de 1 a 5 años $(n=17)$ con un amplio rango desde la primera semana hasta más de 10 años.

Un $57,1 \%(\mathrm{n}=32)$ de los pacientes tenía antecedentes médicos, en el 34,4\% ( $n=11)$ de los casos eran enfermedades cardiovasculares. La Tabla 2 describe los antecedentes médico-quirúrgicos de los pacientes.

La presentación de la obstrucción intestinal se caracterizó por síntomas que en el 73,2\% $(\mathrm{n}=41)$ incluyeron ausencia de deposición y en el 67,9\% $(\mathrm{n}=38)$, emesis y distensión abdominal; la media de síntomas referidos fue 
Tabla 1. Características sociodemográficas de los pacientes con diagnóstico de obstrucción intestinal del HUSJ (2012-2013)

\begin{tabular}{|llcc|}
\hline & Variable & $\mathbf{n}$ & $\%$ \\
\hline Sexo & Mujer & 23 & 41,1 \\
& Hombre & 33 & 58,9 \\
\hline \multirow{2}{*}{ Edad (años) } & Media & 55,2 & \\
& DE & 20,2 & \\
& Mínima & 19 & \\
Procedencia & Máxima & 85 & \\
& Urbana & 34 & 60,7 \\
& Rural & 22 & 39,3 \\
Escolaridad & Ninguna & 7 & 12,5 \\
& Primaria & 18 & 32,1 \\
& Secundaria & 26 & 46,4 \\
& Universitaria & 5 & 8,9 \\
& 1 & 32 & 57,1 \\
Estrato social & 2 & 6 & 10,7 \\
& 2 & 18 & 32,1 \\
& Vinculado & 2 & 3,6 \\
& Subsidiado & 31 & 55,4 \\
Seguridad social & Contributivo & 23 & 41,1 \\
& & &
\end{tabular}

de 2 (rango: 0-4; DE: 0,9). En el examen físico se encontró que $78,6 \%(\mathrm{n}=44)$ de los pacientes tenía peristaltismo; en los signos radiológicos, el $50 \%(\mathrm{n}=28)$ evidenció niveles hidroaéreos y $28,6 \%(n=16)$, ausencia de aire distal. Las pruebas de laboratorio reportaron $26,8 \%(\mathrm{n}=15)$ de leucocitosis y $16,1 \%(n=9)$ de trastorno hidroelectrolítico tipo hipopotasemia. El tiempo transcurrido entre el inicio de los síntomas y la consulta al servicio de urgencias en el $67,9 \%(n=38)$ de los pacientes fue mayor de 24 horas. La Tabla 3 resume las características clínicas.

Todos los pacientes recibieron tratamiento médico, en el $39,3 \%(\mathrm{n}=22)$ de los casos por un período mayor de 48 horas, y el 80,4\% $(n=45)$ respondió a dicho manejo. De los pacientes que requirieron posterior manejo quirúrgico $(\mathrm{n}=11)$, en el procedimiento se evidenció $81,8 \%(\mathrm{n}$ = 9) de bridas, 9,1\% $(\mathrm{n}=1)$ de necrosis y $9,1 \%(\mathrm{n}=1)$ de perforaciones. $\mathrm{Al}$ analizar los desenlaces en función del tipo de tratamiento, se encontró que el tiempo medio de hospitalización fue mucho mayor en los pacientes llevados a cirugía (13,5 frente a 3,6 días), en este grupo se presentó una muerte $y$, al egreso, hubo un porcentaje superior de complicaciones, principalmente de tipo quirúrgico, que fue estadísticamente significativo $(p=0,001$ y $p=0,010$, respectivamente). No se encontró una asociación entre el tiempo transcurrido entre los síntomas y la consulta o
Tabla 2. Antecedentes médico-quirúrgicos de los pacientes incluidos en el estudio

\begin{tabular}{|c|c|c|c|}
\hline \multicolumn{2}{|r|}{ Variable } & $\mathbf{n}$ & $\%$ \\
\hline $\begin{array}{l}\text { Cirugía } \\
\text { abdominal }\end{array}$ & $\begin{array}{l}\text { No } \\
\text { Sí }\end{array}$ & $\begin{array}{c}5 \\
51\end{array}$ & $\begin{array}{r}8,9 \\
91,1\end{array}$ \\
\hline $\begin{array}{l}\text { Tipo de cirugía } \\
\text { abdominal }\end{array}$ & $\begin{array}{l}\text { Cirugía por procesos inflamatorios } \\
\text { Cirugía oncológica } \\
\text { Cirugía por traumatismo } \\
\text { Cirugía vascular } \\
\text { Otras cirugías }\end{array}$ & $\begin{array}{c}24 \\
7 \\
7 \\
1 \\
11\end{array}$ & $\begin{array}{r}48,0 \\
14,0 \\
14,0 \\
2,0 \\
22,0\end{array}$ \\
\hline Cirugía pélvica & $\begin{array}{l}\text { No } \\
\text { Sí }\end{array}$ & $\begin{array}{l}45 \\
11\end{array}$ & $\begin{array}{l}80,4 \\
19,6\end{array}$ \\
\hline $\begin{array}{l}\text { Tipo de cirugía } \\
\text { pélvica }\end{array}$ & $\begin{array}{l}\text { Cesárea } \\
\text { Prostatectomía } \\
\text { Anexo o histerectomía }\end{array}$ & $\begin{array}{l}4 \\
4 \\
3\end{array}$ & $\begin{array}{l}36,4 \\
36,4 \\
27,3\end{array}$ \\
\hline $\begin{array}{l}\text { Cantidad de } \\
\text { procedimientos } \\
\text { quirúrgicos }\end{array}$ & $\begin{array}{l}\text { Media } \\
\text { DE } \\
\text { Mínima } \\
\text { Máxima }\end{array}$ & $\begin{array}{l}1,5 \\
1,2 \\
0 \\
8\end{array}$ & \\
\hline $\begin{array}{l}\text { Tiempo entre la } \\
\text { cirugía y la OIB }\end{array}$ & $\begin{array}{l}\text { Primera semana } \\
\text { Primer mes } \\
\text { Primer año } \\
1-5 \text { años } \\
6-10 \text { años } \\
>10 \text { años }\end{array}$ & $\begin{array}{c}4 \\
4 \\
10 \\
17 \\
8 \\
13\end{array}$ & $\begin{array}{l}7,1 \\
7,1 \\
17,9 \\
30,4 \\
14,3 \\
23,2\end{array}$ \\
\hline $\begin{array}{l}\text { Antecedentes } \\
\text { médicos }\end{array}$ & $\begin{array}{l}\text { No } \\
\text { Sí }\end{array}$ & $\begin{array}{l}24 \\
32\end{array}$ & $\begin{array}{l}42,9 \\
57,1\end{array}$ \\
\hline $\begin{array}{l}\text { Tipo de } \\
\text { antecedentes } \\
\text { médicos }\end{array}$ & $\begin{array}{l}\text { Enfermedad cardiovascular } \\
\text { Enfermedad gastrointestinal } \\
\text { Enfermedad metabólica } \\
\text { Cáncer } \\
\text { Enfermedad neurológica } \\
\text { Otro }\end{array}$ & $\begin{array}{l}11 \\
6 \\
5 \\
3 \\
2 \\
5\end{array}$ & $\begin{array}{c}34,4 \\
18,8 \\
15,6 \\
9,4 \\
6,3 \\
15,6\end{array}$ \\
\hline
\end{tabular}

el tiempo de observación con la presentación de complicaciones ( $p=0,528$ y $p=0,410$, respectivamente). En la Tabla 4 se describen los procedimientos y resultados.

\section{DISCUSIÓN}

Este estudio buscó describir la presentación de la OIB en nuestro centro y cuantificar la respuesta al manejo médico conservador y sus desenlaces basados en las recomendaciones de Bologna (ASBO) para la época (24), y se encontró que más de la mitad de los casos fueron masculinos con una edad media de 55 años, resultado muy similar a lo obtenido en diferentes estudios en Estados Unidos, Honduras y 
Tabla 3. Características clínicas de los pacientes diagnosticados con obstrucción intestinal en el HUSJ (2012-2013)

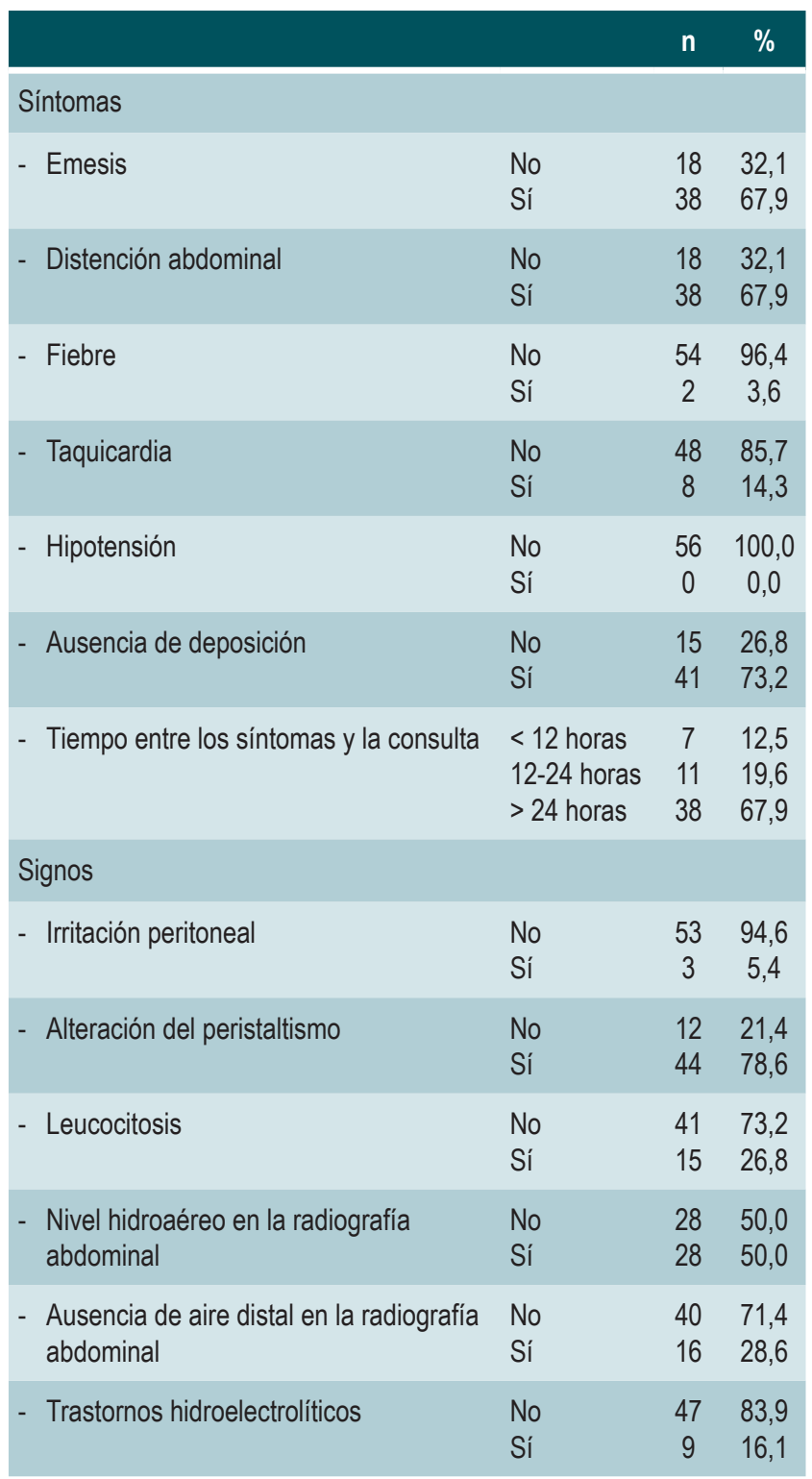

Chile (25-30); pero contrario a lo reportado en otros países de América y España, donde predominaron las mujeres y su edad media fue de 60 años (31-34), lo que podría deberse a las características demográficas de cada territorio.

La causa más común de adherencias peritoneales posoperatorias es una cirugía previa (35), sumado a la cantidad y tipo de procedimientos. Más del $90 \%$ de nuestra población tenía alguna cirugía abdominal previa, principalmente por procesos inflamatorios. Estos datos coinciden con lo reportado en la tesis de López en Perú en su serie de 49 pacientes $(28)$ y otros trabajos $(28,32,36)$; pero contrasta con lo reportado en Centroamérica, donde la OIB se presentó después de 2 cirugías previas y su principal causa (alrededor del $80 \%)$ fue la laparotomía por trauma $(26,33)$.

El tiempo de estos antecedentes fue, en el $30 \%$, en un período de 1 a 5 años y una quinta parte de los pacientes tuvo el procedimiento hace más de 10 años. Este resultado ha sido muy diverso, encontrando en Centroamérica un tiempo medio transcurrido entre los procedimientos y la OIB de 8 años (33); mientras que en Reino Unido se encuentran reportes con formación de adherencias más tempranas, en las que la OIB se produjo dentro de las 4 semanas en el $21 \%$ de los pacientes con antecedentes de cirugía abdominal (37). Las diferencias podrían relacionarse con la etiología del cuadro inicial, el tipo de cirugía previa o la experiencia del cirujano; sin embargo, esta entidad presenta una curva de aparición de difícil predicción y se considera que hay pacientes con tendencia a formar adherencias en mayor proporción que otros ante una misma agresión quirúrgica, que puede deberse a una predisposición particular en cada paciente en relación con la actividad fibrinolítica durante la patogénesis de las adherencias y otros factores (35).

Más de la mitad de los pacientes incluidos en este trabajo tenía antecedentes médicos, principalmente enfermedades cardiovasculares, seguidas de patologías gastrointestinales y metabólicas. Estos resultados son inferiores a los descritos en España y Estados Unidos, donde fueron más frecuentes las enfermedades metabólicas $(31,38)$, lo que podría relacionarse con los estilos de vida.

Con respecto a la presentación clínica, los síntomas predominantes en nuestros pacientes fueron ausencia de deposición y emesis, cuya frecuencia es inversa con respecto a lo reportado en Honduras (26). Asimismo, otros estudios refieren una mayor frecuencia de emesis ( $80 \%)$, seguidos de distención abdominal $(25,28,32,39)$.

En cuanto a los parámetros de laboratorio, se demostró leucocitosis en casi el $30 \%$ de los pacientes y más del $15 \%$ con alteraciones electrolíticas tipo hipocalemia, resultados que superan lo descrito por otros autores $(26,31,34)$. No es posible citar cifras concretas de leucocitosis o cayados, ya que estas presentaban una gran variabilidad entre pacientes, incluso entre aquellos que precisaron resección intestinal. No obstante, las cifras iniciales elevadas de leucocitos por encima de 15000 fue el principal marcador para optar por el tratamiento quirúrgico, tal como lo recomiendan las guías $\operatorname{ASBO}(24,40)$. Se describe la leucocitosis o la leucopenia como indicador de gravedad en la obstrucción intestinal, pero debido posiblemente al corto período de observación de los pacientes, este dato no fue contrastable (31). Una situación similar mencionó Moran, quien afirmó que el recuento de glóbulos blancos puede ser normal o ligeramente elevado en una OIB no complicada, pero los 
Tabla 4. Descripción del tratamiento y sus resultados en la población a estudio

\begin{tabular}{|c|c|c|c|c|c|c|c|c|}
\hline \multicolumn{2}{|c|}{$n=45$} & \multicolumn{2}{|c|}{ Médico } & \multicolumn{2}{|c|}{ Cirugía } & \multirow{2}{*}{$\frac{\text { Total }}{\%}$} & \multicolumn{2}{|c|}{ Valor $p$} \\
\hline & & $\%$ & $n=11$ & $\%$ & $n=56$ & & & \\
\hline Tiempo de manejo médico & $\begin{array}{l}<12 \text { horas } \\
12-24 \text { horas } \\
24-48 \text { horas } \\
>48 \text { horas }\end{array}$ & $\begin{array}{c}4 \\
9 \\
15 \\
17\end{array}$ & $\begin{array}{l}8,9 \\
20,0 \\
33,3 \\
37,8\end{array}$ & $\begin{array}{l}2 \\
2 \\
2 \\
5\end{array}$ & $\begin{array}{l}18,2 \\
18,2 \\
18,2 \\
45,5\end{array}$ & $\begin{array}{c}6 \\
11 \\
17 \\
22\end{array}$ & $\begin{array}{l}10,7 \\
19,6 \\
30,4 \\
39,3\end{array}$ & 0,676 \\
\hline Hallazgos quirúrgicos & $\begin{array}{l}\text { Bridas } \\
\text { Necrosis } \\
\text { Perforación }\end{array}$ & & & $\begin{array}{l}9 \\
1 \\
1\end{array}$ & $\begin{array}{c}81,8 \\
9,1 \\
9,1\end{array}$ & $\begin{array}{l}9 \\
1 \\
1\end{array}$ & $\begin{array}{l}81,8 \\
9,1 \\
9,1\end{array}$ & - \\
\hline Complicaciones & $\begin{array}{l}\text { No } \\
\text { Médicas } \\
\text { Quirúrgicas }\end{array}$ & $\begin{array}{c}41 \\
4 \\
0\end{array}$ & $\begin{array}{l}91,1 \\
8,9 \\
0,0\end{array}$ & $\begin{array}{l}6 \\
2 \\
3\end{array}$ & $\begin{array}{l}54,5 \\
18,2 \\
27,3\end{array}$ & $\begin{array}{c}47 \\
6 \\
3\end{array}$ & $\begin{array}{c}83,9 \\
10,7 \\
5,4\end{array}$ & 0,001 \\
\hline Tiempo total de hospitalización & $\begin{array}{l}\text { Media } \\
\text { DE }\end{array}$ & $\begin{array}{l}3,6 \\
3,2\end{array}$ & & $\begin{array}{l}13,5 \\
10,6\end{array}$ & & $\begin{array}{l}5,5 \\
6,7\end{array}$ & & 0,010 \\
\hline Estado al egreso & $\begin{array}{l}\text { Muerto } \\
\text { Vivo con complicaciones } \\
\text { Vivo sin complicaciones }\end{array}$ & $\begin{array}{c}0 \\
4 \\
41\end{array}$ & $\begin{array}{c}0,0 \\
8,9 \\
91,1\end{array}$ & $\begin{array}{l}1 \\
2 \\
8\end{array}$ & $\begin{array}{c}9,1 \\
18,2 \\
72,7\end{array}$ & $\begin{array}{c}1 \\
6 \\
49\end{array}$ & $\begin{array}{c}1,8 \\
10,7 \\
87,5\end{array}$ & 0,076 \\
\hline
\end{tabular}

recuentos altos (más de 15000 glóbulos blancos) o muy bajos (menos de 4000) son sospechosos y deben alertar a los médicos sobre la posibilidad de isquemia intestinal (41).

La prueba de imagen básica y diagnóstica en todos los cuadros de obstrucción intestinal en nuestro estudio fue la radiografía de abdomen, en la que se evidenciaron niveles hidroaéreos en un $50 \%$ y ausencia de aire distal en el 28,6\%; lo que difiere de otros estudios $(30,32,34)$ en los que refieren que la tomografía axial computarizada (TAC) fue el examen imagenológico más utilizado para establecer la causa, detectar signos de complicaciones y definir el tratamiento. La radiografía simple será en principio la modalidad de primera línea diagnóstica por su bajo costo y su alta disponibilidad $(24,40)$; sin embargo, la TAC es el estándar de oro por su alta sensibilidad y especificidad $(90 \%-96 \%)(39,42)$.

Los signos vitales son importantes en la apreciación diagnóstica y pronóstica, nuestros pacientes presentaron taquicardia $(14,3 \%)$ y fiebre $(3,6 \%)$, en un porcentaje menor respecto a lo reportado en estudios del centro y norte de América, con un $40 \%$ de taquicardia $(26,34)$. En la literatura revisada no se informan a menudo dichos síntomas, debido a que en la mayoría de los estudios se utilizaron estos parámetros como criterios de exclusión al ser subjetivos de estrangulación o perforación intestinal, lo que implica un tratamiento quirúrgico inmediato. En Asia, la obstrucción simple se define como la presencia de solo adherencias, mientras que la obstrucción complicada incluye la presencia de gangrena o estrangulación. En pacientes con obs- trucción complicada, la fiebre estaba presente en 18 (67\%) casos y leucocitosis (más de $15000 / \mathrm{mm}$ ) en 20 (74\%) casos. Ninguno de los pacientes con obstrucción simple tuvo estos hallazgos (43).

El tiempo transcurrido entre el inicio de los síntomas y la consulta al servicio de urgencias fue mayor de 24 horas en dos tercios de los pacientes, similar a lo reportado por otros estudios que refirieron 2-3 días de evolución en la sintomatología (26). La respuesta al manejo conservador de nuestra población fue exitoso en el $80 \%$ y, de ellos, el $90 \%$ egresó sin complicaciones, superior a lo descrito en otras poblaciones en las que este resultado varió entre el $20 \%$ y el $60 \%(27,29,31,32,34,36,43,44)$. Estas cifras podrían relacionarse con el tipo de cirugía que dio origen a la OIB, en las que predominaron los procesos inflamatorios, que sugieren intervenciones menos invasivas y con menor manipulación de la cavidad abdominal.

Por su parte, la obstrucción intestinal representa el $30 \%$ $41 \%$ de todos los casos que requieren cirugía adicional (7), frecuencias que duplicanlo descrito en este estudio $(<20 \%)$; y el principal hallazgo quirúrgico son las adherencias (44), concordante con lo encontrado en nuestros pacientes (> $80 \%)$; pero este dato supera otros reportes $(<50 \%)(27$, $29,31,32,34,44)$. La estancia hospitalaria promedio de los pacientes tratados de forma conservadora fue de 3 días, tiempo que se cuadruplicó en los manejados quirúrgicamente con diferencias estadísticamente significativas ( $p$ $=0,010)$; esta temporalidad concuerda con otros autores 
cuyos pacientes llevados a cirugía alcanzan una estancia media de 10 días (32-34, 40, 45). Esto puede relacionarse con la alta frecuencia de complicaciones ( $>40 \%$ ) reportadas en este grupo, lo que también fue significativo ( $p=$ $0,010)$. En general, la mortalidad de la OIB es del $2 \%$ tanto en nuestra población como en la de diferentes estudios, presentándose exclusivamente en los pacientes llevados a cirugía $(32,44)$.

Entre las limitaciones encontradas en este estudio estuvo no contar en el centro asistencial con la TAC para definir con mayor precisión el diagnóstico; no obstante, se deben considerar los recursos disponibles en el contexto y tiempo en el que se desarrolló este estudio y que la priorización de este tipo de imagen se presentó en las guías ASBO del 2017 (42). Igualmente, el período de recolección de la información de 1 año limitó la cantidad de pacientes para establecer significancia estadística y no hacer seguimiento posterior al alta para determinar las recidivas o mortalidad. Entre las fortalezas se encuentran ser el primer estudio de este tipo en el suroccidente colombiano, que aplica guías de práctica clínica internacionales y que obtuvo resultados positivos que superan los reportes mundiales para este evento.

Como conclusión, se puede mencionar que la OIB posoperatoria afecta mayormente a los varones, el diagnóstico debe considerar el conjunto de antecedentes quirúrgicos, síntomas, exámenes paraclínicos e imágenes radiológicas. El manejo médico conservador debe ser considerado como el tratamiento inicial de esta patología, debido a que presenta una alta tasa de respuesta y que, adicionalmente, disminuye las complicaciones y la estancia hospitalaria.

\section{Fuente de financiación}

Ninguna.

\section{REFERENCIAS}

1. Duron JJ. Postoperative intraperitoneal adhesion pathophysiology. Colorectal Dis. 2007;9(s2):14-24. https://doi.org/10.1111/j.1463-1318.2007.01343.x

2. Ellis $\mathrm{H}$. The magnitude of adhesion related problems. Ann Chir Gynaecol. 1998;87(1):9-11.

3. Al-Jaroudi D, Tulandi T. Adhesion prevention in gynecologic surgery. Obstet Gynecol Surv. 2004;59(5):360-7. https://doi.org/10.1097/00006254-200405000-00024

4. Ozel H, Avsar FM, Topaloglu S, Sahin M. Induction and assessment methods used in experimental adhesion studies. Wound Repair Regen. 2005;13(4):358-64. https://doi.org/10.1111/j.1067-1927.2005.130402.x

5. Boland GM, Weigel RJ. Formation and prevention of postoperative abdominal adhesions. J Surg Res. 2006; 132(1):3-12. https://doi.org/10.1016/j.jss.2005.12.002

6. Ouaïssi M, Gaujoux $S$, Veyrie N, Denève E, Brigand C, Castel B, Duron JJ, Rault A, Slim K, Nocca D. Postoperative adhesions after digestive surgery: their incidence and prevention: review of the literature. J Visc Surg. 2012;149(2):e104-14. https://doi.org/10.1016/j.jviscsurg.2011.11.006

7. Parker MC, Ellis H, Moran BJ, Thompson JN, Wilson MS, Menzies D, McGuire A, Lower AM, Hawthorn RJ, O’Briena F, Buchan S, Crowe AM. Postoperative adhesions: ten-year follow-up of 12,584 patients undergoing lower abdominal surgery. Colon Rectum. 2001;44(6):822-9.

https://doi.org/10.1007/BF02234701
8. Sykes PA, Schofield PF. Early postoperative small bowel obstruction. Br J Surg. 1974;61(8):594-600. https://doi.org/10.1002/bjs.1800610803

9. Köveker GB, Coerper S, Gottwald T, Flesch I, Becker H-D. The role of wound healing in the formation of peritoneal adhesions. En: Treutner KH, Schumpelick V (editores). Peritoneal adhesions. Springer; 1997. p. 23-8. https://doi.org/10.1007/978-3-642-60433-1_3

10. Ansari P. Intestinal Obstruction - Gastrointestinal Disorders [Internet]. Merck Manuals Professional Edition. 2020 [citado 13 de diciembre de 2020]. Disponible en: https://www.merckmanuals.com/professional/ gastrointestinal-disorders/acute-abdomen-and-surgicalgastroenterology/intestinal-obstruction

11. Schnüriger B, Barmparas G, Branco BC, Lustenberger T, Inaba K, Demetriades D. Prevention of postoperative peritoneal adhesions: a review of the literature. Am J Surg. 2011;201(1):111-21. https://doi.org/10.1016/j.amjsurg.2010.02.008

12. Tulandi T, Collins JA, Burrows E, Jarrell JF, McInnes RA, Wrixon W, Simpson CW.Treatment-dependent and treatment-independent pregnancy among women with periadnexal adhesions. Am J Obstet Gynecol. 1990;162(2):354-7. https://doi.org/10.1016/0002-9378(90)90384-J

13. Marana R, Rizzi M, Muzii L, Catalano GF, Caruana P, Mancuso S. Correlation between the American Fertility Society classifications of adnexal adhesions and distal tubal occlusion, salpingoscopy, and reproductive outcome in 
tubal surgery. Fertil Steril. 1995;64(5):924-9. https://doi.org/10.1016/S0015-0282(16)57903-5

14. Marana R, Catalano GF, Muzii L, Caruana P, Margutti F, Mancuso $S$. The prognostic role of salpingoscopy in laparoscopic tubal surgery. Hum Reprod. 1999;14(12):2991-5. https://doi.org/10.1093/humrep/14.12.2991

15. Milingos S, Kallipolitis G, Loutradis D, Liapi A, Mavrommatis K, Drakakis P, Tourikis J, Creatsas G, Michalas S. Adhesions: laparoscopic surgery versus laparotomy. Ann N Acad Sci. 2000;900(1):272-85. https://doi.org/10.1111/j.1749-6632.2000.tb06239.x

16. Vrijland W, Jeekel J, Van Geldorp H, Swank D, Bonjer H. Abdominal adhesions: intestinal obstruction, pain, and infertility. Surg Endosc. 2003;17(7):1017-22. https://doi.org/10.1007/s00464-002-9208-9

17. Luijendijk RW, de Lange DC, Wauters CC, Hop WC, Duron JJ, Pailler JL, Camprodon BR, Holmdahl L, van Geldorp HJ, Jeekel J. Foreign material in postoperative adhesions. Ann Surg. 1996;223(3):242-8. https://doi.org/10.1097/00000658-199603000-00003

18. Parker MC, Wilson MS, Menzies D, Sunderland G, Clark DN, Knight AD, Crowe AM; Surgical and Clinical Adhesions Research (SCAR) Group. The SCAR-3 study: 5-year adhesion-related readmission risk following lower abdominal surgical procedures. Colorectal Dis. 2005; $7(6): 551-8$. https://doi.org/10.1111/j.1463-1318.2005.00857.x

19. Nunobe S, Hiki N, Fukunaga T, Tokunaga M, Ohyama S, Seto $Y$, Yamaguchi T. Previous laparotomy is not a contraindication to laparoscopy-assisted gastrectomy for early gastric cancer. World J Surg. 2008;32(7):1466-72. https://doi.org/10.1007/s00268-008-9542-8

20. Ward BC, Panitch A. Abdominal adhesions: current and novel therapies. J Surg Res. 2011;165(1):91-111. https://doi.org/10.1016/j.jss.2009.09.015

21. Glancy DG. Intestinal obstruction. Surg Oxf. 2014;32(4):204-11. https://doi.org/10.1016/j.mpsur.2014.01.004

22. Coleman MG, McLain AD, Moran MB. Impact of previous surgery on time taken for incision and division of adhesions during laparotomy. Colon Rectum. 2000;43(9):1297-9. https://doi.org/10.1007/BF02237441

23. Van Der Krabben AA, Dijkstra FR, Nieuwenhuijzen $M$, Reijnen M, Schaapveld M, Van Goor H. Morbidity and mortality of inadvertent enterotomy during adhesiotomy. Br J Surg. 2000;87(4):467-71. https://doi.org/10.1046/j.1365-2168.2000.01394.x

24. Catena F, Di Saverio S, Kelly MD, Biffl WL, Ansaloni L, Mandalà V, Velmahos GC, Sartelli M, Tugnoli G, Lupo $\mathrm{M}$, Mandalà S, Pinna AD, Sugarbaker PH, Van Goor H, Moore EE, Jeekel J. Bologna Guidelines for Diagnosis and Management of Adhesive Small Bowel Obstruction (ASBO): 2010 Evidence-Based Guidelines of the World Society of Emergency Surgery. World J Emerg Surg. 2011 Jan 21;6:5. https://doi.org/10.1186/1749-7922-6-5
25. Luque García MJ. Factores pronósticos relacionados con la cirugía de la oclusión intestinal [Internet]. Madrid: Universidad Complutense de Madrid; 2014 [citado el 14 de enero de 2019]. Disponible en: https://eprints.ucm. es/33035/1/T36359.pdf

26. Canales Peña RC, Cortés Cardona CM, Cabrera Interiano H. Obstrucción Intestinal por Bridas/Adherencias: Proporción y Evolución del Manejo Conservador. Arch Med. 2017;13(3):1-6.

27. Quezada S Nicolás, León F Felipe, Llera KJuan de la, Funke H Ricardo, Gabrielli N Mauricio, Crovari E Fernando et al . TRATAMIENTO LAPAROSCÓPICO DE LA OBSTRUCCIÓN INTESTINAL POR BRIDAS. Rev Chil Cir [Internet]. 2014 Sep [citado 2020 Dic 13] ; 66 ( 5 ): 437-442. Disponible en: https://scielo. conicyt.cl/scielo.php?script=sci_arttext\&pid=S071840262014000500007\&lng=es. http://dx.doi. org/10.4067/S0718-40262014000500007.

28. López Lorenzo RE. Características clínico epidemiológicas de pacientes intervenidos por obstrucción intestinal por bridas y adherencias en el hospital regional docente clínico quirúrgico Daniel Alcides Carrión Huancayo, durante el año 2016 [Internet]. Perú: Universidad Nacional del Centro del Perú; 2017 [citado el 14 de enero de 2019]. Disponible en: http://repositorio.uncp.edu.pe/bitstream/ handle/UNCP/1548/CARACTER\%C3\%8DSTICAS\%20 CL\%C3\%8DNICO\%20EPIDEMIOL\%C3\%93GICAS\%20 DE\%20PACIENTES\%20INTERVENIDOS\%20POR\%20 OBSTRUCCI\%C3\%93N\%2OINTESTINAL\%20 POR\%20.pdf?sequence $=1$ \&isAllowed $=y$

29. García Rengifo REO. Obstrucción intestinal asociada a cirugía de urgencia en el adulto mayor, Hospital II-2 Tarapoto 2015-2016. [Internet]. Perú: Universidad Nacional de San Martín-Tarapoto; 2018 [citado el 14 de enero de 2019]. Disponible en: http://repositorio.unsm. edu.pe/handle/11458/2611

30. Weaver JL, Barnett RE, Patterson DE, Ramjee VG, Riedinger E, Younga J, Sepulveda EA, Keskey RC, Cheadle WG. Large-bowel disease presenting as small-bowel obstruction is associated with a poor prognosis. Am J Emerg Med. 2016;34(3):477-9. https://doi.org/10.1016/j.ajem.2015.12.008

31. Enríquez Sánchez S. Análisis de la obstrucción intestinal en pacientes mayores de 50 años [Internet]. Granada: Universidad de Granada; 2007 [citado el 14 de enero de 2019]. Disponible en: http://digibug.ugr.es/bitstream/ handle $/ 10481 / 1725 / 17243750$.pdf;jsessionid $=876792 \mathrm{E} 2$ 94C9233B7366CF4F5BBC8D71 ? sequence $=1$

32. Csendes A, Pereira P, Zamorano M, Arratia I, Gonzalez J, Carriel F. Tratamiento médico o quirúrgico de la obstrucción intestinal alta. Rev Chil Cir. 2016;68(3):227-32. https://doi.org/10.1016/j.rchic.2014.08.001

33. Garibay-González F, Navarrete-Arellano M, MorenoDelgado F, Salinas-Hernández EL, Rodríguez-Ayala E, Cleva-Villanueva G. Incidencia de la obstrucción intestinal 
por adherencias postoperatorias en el Hospital Central Militar. Factores de riesgo asociados. Rev Sanid Milit Mex. 2017;71(6):534-44.

34. Zielinski MD, Eiken PW, Bannon MP, Heller SF, Lohse CM, Huebner M, Sarr MG. Small bowel obstruction-who needs an operation? A multivariate prediction model. World J Surg. 2010;34(5):910-9. https://doi.org/10.1007/s00268-010-0479-3

35. Correa-Rovelo JM, Villanueva-López GC, MedinaSantillan R, Carrillo-Esper R, Díaz-Girón-Gidi A. Obstrucción intestinal secundaria a formación de adherencias postoperatorias en cirugía abdominal. Revisión de la literatura. Cir Cir. 2015;83(4):345-51. https://doi.org/10.1016/j.circir.2015.05.024

36. Millet I, Ruyer A, Alili C, Curros Doyon F, Molinari N, Pages E, Zins M, Taourel P.Adhesive small-bowel obstruction: value of CT in identifying findings associated with the effectiveness of nonsurgical treatment. Radiology. 2014;273(2):425-32. https://doi.org/10.1148/radiol.14132872

37. Menzies D, Ellis H. Intestinal obstruction from adhesions--how big is the problem? Ann R Coll Surg Engl. 1990;72(1):60-3.

38. Mavros MN, Velmahos GC, Lee J, Larentzakis A, Kaafarani HM. Morbidity related to concomitant adhesions in abdominal surgery. J Surg Res. 2014;192(2):286-92. https://doi.org/10.1016/j.jss.2014.07.044

39. Rosero Vallejos YL. Factores predictores en tomografía computarizada de necesidad de cirugía en pacientes con obstrucción del intestino delgado: Estudio de cohorte (mayo de 2016 hasta mayo de 2018) [Internet]. Bogotá: Universidad Nacional de Colombia; 2018 [citado el 14 de enero de 2019]. Disponible en: https: / / repositorio.unal. edu.co/bitstream/handle/unal/68650/53009551.2018. pdf? sequence $=1$ \&isAllowed $=y$

40. Di Saverio S, Coccolini F, Galati M, Smerieri N, Biffl WL, Ansaloni L, Tugnoli G, Velmahos GC, Sartelli M, Bendinelli C, Fraga GP, Kelly MD, Moore FA, Mandalà V, Mandalà S, Masetti M, Jovine E, Pinna AD, Peitzman AB,
Leppaniemi A, Sugarbaker PH, Goor HV, Moore EE, Jeekel J, Catena F. Bologna guidelines for diagnosis and management of adhesive small bowel obstruction (ASBO): 2013 update of the evidence-based guidelines from the world society of emergency surgery ASBO working group. World J Emerg Surg. 2013;8(1):42. https://doi.org/10.1186/1749-7922-8-42

41. Moran BJ. Adhesion-related small bowel obstruction. Colorectal Dis. 2007;9(2):39-44. https://doi.org/10.1111/j.1463-1318.2007.01347.x

42. Ten Broek RPG, Krielen P, Di Saverio S, Coccolini F, Biffl WL, Ansaloni L, Velmahos GC, Sartelli M, Fraga GP, Kelly MD, Moore FA, Peitzman AB, Leppaniemi A, Moore EE, Jeekel J, Kluger Y, Sugrue M, Balogh ZJ, Bendinelli C, Civil I, Coimbra R, De Moya M, Ferrada P, Inaba K, Ivatury R, Latifi R, Kashuk JL, Kirkpatrick AW, Maier R, Rizoli S, Sakakushev B, Scalea T, Søreide K, Weber D, Wani I, AbuZidan FM, De'Angelis N, Piscioneri F, Galante JM, Catena $\mathrm{F}$, van Goor $\mathrm{H}$. Bologna guidelines for diagnosis and management of adhesive small bowel obstruction (ASBO): 2017 update of the evidence-based guidelines from the world society of emergency surgery ASBO working group. World J Emerg Surg. 2018;13:24. https://doi.org/10.1186/s13017-018-0185-2

43. Shih S-C, Jeng K-S, Lin S-C, Kao C-R, Chou S-Y, Wang $\mathrm{H}-\mathrm{Y}$, Chang WH, Chu CH, Wang TE. Adhesive small bowel obstruction: how long can patients tolerate conservative treatment? World J Gastroenterol. 2003;9(3):603-5. https://doi.org/10.3748/wjg.v9.i3.603

44. Springer JE, Bailey JG, Davis PJ, Johnson PM. Management and outcomes of small bowel obstruction in older adult patients: a prospective cohort study. Can J Surg. 2014;57(6):379-84. https://doi.org/10.1503/cjs.029513

45. Tavangari FR, Batech M, Collins JC, Tejirian T. Small bowel obstructions in a virgin abdomen: is an operation mandatory? Am Surg. 2016;82(10):1038-42. https://doi.org/10.1177/000313481608201040 